at Mt. Wilson has shown that the opacity of the vapour of an exploded iron wire under certain conditions is such that light is cut off in a distance not greater than a few centimetres. Application of the above equation for $K$ indicates that the absorption of radiant energy by the free electrons in the doubly ionised iron vapour produces an opacity of this order of magnitude. Thus, estimating $T$ as 20,000 degrees absolute, $i$ as $2, A$ as $5 \times 10^{-8} \mathrm{~cm}$. (doubtful), and $p /(\mathrm{I}+i)$, the partial pressure of the iron ions, as 20 atmospheres (doubtful), $K$ comes out as $\mathrm{I} \cdot 7$ for $\lambda=6 \times 10^{-5} \mathrm{~cm}$. The electrical conductivity of the vapour theoretically is $\mathrm{r} / \mathrm{I} 5$ ooth that of metallic copper.

Application of the equation for $K$ to conditions in the outer regions of the sun, employing Saha's theory to calculate the ionisation as a-function of the unknown gas pressure, makes it seem probable that at a depth in the sun where the pressure is as great as o.or atmosphere the ionised gas is sufficiently opaque to cut off radiation from farther down. This is, then, indicated as the approximate pressure in the solar photosphere; and pressures in the solar atmosphere are much lower. Thus the sharpness of the Fraunhofer lines may be explained. I hope soon to publish these results in detail. The astrophysical importance of the matter is obvious.

Naturally it will require a great deal of study to develop more than a rough theory of the opacity of an ionised gas. Radiation is selectively scattered by bound electrons; it is non-selectively scattered by free electrons ; and it is absorbed by free electrons. The part played by bound electrons in absorbing radiation (that is, in transforming it to heat) seems at present far from understood. Prof. Eddington's recent discussion (Observatory, December 1922) of the absorption of radiation by quanta in the deep interior of stars perhaps opens a new line of attack on the general problem.

Princeton University Observatory, Princeton, New Jersey, January 8 .

\section{The High Temperature of the Upper Atmosphere} as an Explanation of Zones of Audibility.

THE work of Lindemann and Dobson on the theory of meteors, ${ }^{1}$ with the remarkable conclusion that the temperature of the atmosphere at heights such as 80 kilometres is about the same as that near the earth's surface, will be far-reaching in its influence. May I be allowed to point out that one of the phenomena for which an explanation will probably be provided is the occurrence of zones of audibility and zones of silence, surrounding the scenes of great explosions.

If, as Lindemann and Dobson find, temperature increases rather rapidly at about 60 kilometres, then sound waves penetrating that region will be refracted back to earth, the comparatively rapid curvature of the sound rays making the phenomenon almost equivalent to reflection as is the case with the light rays which occasion mirage.

If we assume a sharp transition of temperature from $220^{\circ} \mathrm{A}$. to $280^{\circ} \mathrm{A}$. we find a refractive index for sound rays passing from the lower level to the upper of $\sqrt{280 / 220}$ or I.I3. Total reflection takes place with an angle of incidence $62^{\circ}$, and if the reflection is at 60 kilometres the minimum radius for the outer zone of audibility is $2 \times 60 \times \tan 62^{\circ}$ or I 55 kilometres.

This rough estimate is of the right order of magnitude, as may be seen by comparison with the most recent

'A Theory of Meteors, and the Density and Temperature of the Outer Atmosphere to which it leads. F. A. Lindemann and G. M. B. Dobson (Royal Society Proceedings, vol. I02, I922, p. 4rr). example, the Oldbroek Explosion of October 28, I922, for which the corresponding limit is stated to have been " about I8o or $200 \mathrm{~km}$." (NAtURE, January 6, p. 33).

There should be no great difficulty in adapting the theories worked out by von dem Borne and de Quervain to the new hypothesis. The drift of meteor trails shows that there is considerable horizontal motion of the atmosphere at such heights as 60 kilometres, and this motion will have to be taken into account. It is not unlikely that monsoonal changes in the upper winds produce the seasonal variation in the direction of audibility which was so noticeable during the war. The number of known observations of meteor trails is too small ( $c f$. Meteorological Magazine, vol. 56, p. 292, I92 I) to throw any light on this question.

Further progress in our knowledge of the temperature of the outer atmosphere and of its motion would be made if Prof. Goddard could send up his rockets. The times of passage of the sound waves from the bursting rockets would give immediate information as to the temperature of the air. Perhaps it would be more practicable to use a "Big Bertha" to send up a bursting shell. Mr. Denning could say, no doubt, whether there are any instances in which the disruption of a meteor has been heard and the time interval between sight and sound has been recorded.

With regard to the theory suggested by Lindemann and Dobson in explanation of the high temperature of the outer atmosphere, it should be pointed out that the atmosphere is only exposed to solar radiation during the day-time. It would seem that the equation by which the authors determine the steady temperature should be modified considerably. Annual variation in the temperature of these outer layers of the atmosphere is to be anticipated ; it is not unlikely that examination of the statistics regarding meteors will reveal it. According to the theory meteors should reach much lower levels in winter than in summer.

6 Addison Road, Bedford Park, W.4.

\section{Fixation of Nitrogen by Plants.}

IN NATURE of January 20, p. 95, reference is made to an announcement in Science by Lipman and Taylor that they have proved conclusively the fixation of atmospheric nitrogen by the wheat plant. Should the detailed evidence, when available, show that their claim is well founded, it should not be forgotten that similar results on other plants were obtained in this country some little time ago by the late Prof. Benjamin Moore and his co-workers. In two communications to the Royal Society (Proc. Roy. Soc., B, vols. 91 and 92, I920), he argues strongly in favour of such fixation, supporting his views by convincing experimental proof on both fresh-water and marine algæ. The work was incorporated in his book "Biochemistry" (I92I), and in the Hugo Müller memorial lecture delivered before the Chemical Society in June of that year-one of his last public utterances - he reiterates in the strongest language his belief, founded upon no inconsiderable amount of experimental work, "that both the lower and higher plants do build up nitrites and nitrates and form organic nitrogenous compounds from the free nitrogen of the atmosphere."

I may say that in their article in Science Messrs. Lipman and Taylor give references to Moore's work as to that of other previous observers.

\footnotetext{
Biochemical Department, University of Oxford, January 24.
}

EDWARD WHITLEY. 